\title{
L'IMPORTANZA DEL FOLLOW-UP PSICOLOGICO E NUTRIZIONALE DOPO LA CHIRURGIA BARIATRICA: REVISIONE DELLA
}

\section{LETTERATURA}

\section{ARTICOLO ORIGINALE}

DENDASCK, Carla Viana ${ }^{1}$, SANTOS, Rogério Bongestab dos ${ }^{2}$, SANTOS, Vitor Maia $^{3}$, ANDRADE, Tadeu Uggere de ${ }^{4}$, PFLUG, Adriano Ribeiro Meyer ${ }^{5}$

DENDASCK, Carla Viana. Et al. L'importanza del follow-up psicologico e nutrizionale dopo la chirurgia bariatrica: revisione della letteratura. Revista Científica Multidisciplinar Núcleo do Conhecimento. Anno. 06, Ed. 10, Vol. 01, pp. 20-44. Ottobre 2021. ISSN: 2448-0959, Link di accesso: https://www.nucleodoconhecimento.com.br/psicologia-it/follow-up-psicologico, DOI: 10.32749/nucleodoconhecimento.com.br/psicologia-it/follow-up-psicologico

\footnotetext{
${ }^{1}$ Teologo, PhD in Psicoanalisi Clinica. Si occupa da 15 anni di Metodologia Scientifica (Metodo di Ricerca) in Orientamento alla Produzione Scientifica per Master e Dottorandi. Specialista in Ricerche di Mercato e Ricerche Sanitarie Dottorando in Comunicazione e Semiotica (PUC SP).

${ }^{2}$ Laureato in Medicina presso la Scuola di Scienze di Santa Casa de Misericórdia de Vitória - EMESCAM. Residenza medica in chirurgia generale presso l'Ospedale Santa Casa de Misericórdia de Vitória (HSCMV). Postlaurea in Terapia Nutrizionale presso EMESCAM. Laureato in Nutrologia presso la Facoltà di Scienze Mediche di Santa Casa de Misericórdia di San Paolo (FCMSCM-SP/ABRAN). Specialista in Nutrologia presso l'Associazione Brasiliana di Nutrologia (ABRAN). Specialista in Terapia Nutrizionale presso la Società Brasiliana di Nutrizione Parenterale ed Enterale (SBNPE/BRASPEN).

${ }^{3}$ Psichiatra. Specialista in Psichiatria e Psichiatria Forense presso l'Associazione Brasiliana di Psichiatria (ABP). Dottore presso il Ministero dell'Economia (ME). Studente magistrale qualificato in Scienze farmaceutiche presso l'Università di Vila Velha (UVV). Vice Segretario Direttore dell'Associazione Psichiatrica Espírito Santo (APES). Segretario della Commissione Psichiatria del Lavoro ABP. Professore nel Corso di Perfezionamento in Psichiatria presso l'Università di Vila Velha (UVV). Laurea Magistrale in Medicina del Lavoro e Laurea Magistrale in Perizie Mediche.

${ }^{4}$ Dottorato in Scienze Fisiologiche - Università Federale dell'Espírito Santo, UFES. Master in International Management - Steinbeis University Berlin, STW-SIBE. Laurea Magistrale in Scienze Fisiologiche - Università Federale dell'Espírito Santo, UFES. Specializzazione in Gastronomia Funzionale - San Paolo Method College, FAMESP. Laurea in Farmacia - Facoltà di Farmacia e Biochimica di Espírito Santo, FAFABES.

${ }^{5}$ Dottore di FMUSP. Laureato in Medicina presso la Facoltà di Medicina dell'Università di San Paolo (FMUSP). Residenza medica in Chirurgia Generale e Chirurgia Generale Avanzata presso Hospital das Clinicas, Facoltà di Medicina, Università di San Paolo (HC-FMUSP). Chirurgo Generale del Collegio Brasiliano di Chirurgia (CBC), Chirurgo dell'apparato digerente del Collegio Brasiliano di Chirurgia Digestiva (CBCD). Chirurgia bariatrica (SBCBM) Assistente medico della Clinica chirurgica Divisione III dell'Istituto centrale di Hospital das Clínicas presso FMUSP.
}

RC: 98482

Disponibile in: https://www.nucleodoconhecimento.com.br/psicologia-it/follow-uppsicologico 


\section{RIEPILOGO}

L'obesità è una malattia cronica multifattoriale. II paziente può presentare diverse condizioni cliniche ad esso associate. II quadro clinico è complesso, il che può ostacolare l'uso di trattamenti e metodi convenzionali. La chirurgia bariatrica è un trattamento efficace a lungo termine per questa condizione. Sulla base di una revisione della letteratura, l'articolo mira a discutere l'importanza del follow-up psicologico e nutrizionale post-bariatrico. La domanda fondamentale che sta alla base di questo studio è: in che modo le strategie tipiche nel periodo postoperatorio possono contribuire all'individuo sottoposto a intervento chirurgico per dare maggiore importanza alla sua dieta e alla sua salute mentale? La strategia di trattamento più efficace è stata la chirurgia bariatrica, tuttavia, la valutazione multidisciplinare che segna lo stato biologico, sociale e psicologico del paziente è fondamentale, poiché i cambiamenti psicopatologici sono comuni agli individui obesi. Sebbene la chirurgia bariatrica contribuisca al miglioramento della qualità della vita dei pazienti, è necessario prestare maggiore attenzione al trattamento postoperatorio. Questi individui dovrebbero essere incoraggiati a continuare con il follow-up nutrizionale e psicologico nel periodo postoperatorio, in modo che sia possibile prevenire possibili complicazioni a medio e lungo termine.

Parole chiave: Obesità, Salute mentale, Monitoraggio nutrizionale, Bariatrico.

\section{INTRODUZIONE}

L'obesità è un'epidemia in aumento e quindi facilita lo sviluppo di comorbidità, come il diabete e l'ipertensione arteriosa sistemica (RODRIGUES et al., 2017). Questo raggiunge scala globale, circa due miliardi di persone sono obese 0 in sovrappeso. Nel 2008, è stato stimato che circa il $43 \%$ della popolazione adulta era obesa, tuttavia, questo numero è aumentato, raggiungendo il 54\% nel 2015 (RODRIGUES et al., 2017). In Brasile, è stato identificato che, secondo i dati del Sistema di sorveglianza dei fattori di rischio e protettivi per le malattie croniche di Telephone

RC: 98482

Disponibile in: https://www.nucleodoconhecimento.com.br/psicologia-it/follow-uppsicologico 
Survey, il $53 \%$ della popolazione adulta era considerato sovrappeso e il $18,9 \%$ obeso (BRASIL, 2017). Con l'aumento dell'obesità, il numero di interventi chirurgici bariatrici è aumentato di conseguenza, come sottolineato dalla Società brasiliana di chirurgia bariatrica e metabolica (SBCBM, 2017). II Brasile è il secondo paese al mondo per numero di interventi chirurgici bariatrici eseguiti ogni anno. Rispetto al 2015, c'è stato un aumento del $7,5 \%$ degli interventi chirurgici eseguiti (SBCBM, 2017). La perdita di peso dopo l'intervento chirurgico riflette un significativo miglioramento delle comorbidità, come diabete, dislipidemia, ipertensione e apnea del sonno (BUCHWALD et al., 2004). Tuttavia, va sottolineato che il trattamento chirurgico è solo una parte correlata al trattamento integrale dell'obesità e il follow-up nutrizionale e psicologico dopo l'intervento chirurgico è di fondamentale importanza. Pertanto, si dovrebbe investire in strategie per promuovere la salute e l'assistenza clinica longitudinale (BRASIL, 2017). II paziente che mira a eseguire l'operazione deve soddisfare alcuni requisiti, indipendentemente dalla tecnica chirurgica, come BMI superiore a $40 \mathrm{~kg} / \mathrm{m}^{2}$ o BMl superiore a $35 \mathrm{~kg} / \mathrm{m}^{2}$ in presenza di comorbidità correlate al sovrappeso (SBCBM, 2006). L'elenco delle comorbilità è stato ampliato ed è stata contemplata una porzione più ampia della popolazione (BRASIL, 2017).

Ogni paziente sottoposto a questo tipo di intervento chirurgico deve essere sottoposto a un follow-up multidisciplinare. L'obiettivo è la valutazione, l'orientamento e lo zelo per la relazione team-paziente, in quanto questo è un modo per promuovere l'efficacia del trattamento nel periodo postoperatorio (GORDON; KAIO; SALLET, 2011). II nutrizionista svolge un ruolo vitale nei periodi pre e postoperatorio. La consulenza nutrizionale nel periodo preoperatorio è intrinsecamente correlata all'aumento del potenziale di successo nel periodo postoperatorio (CRUZ, 2004). Uno studio relativo alla recidiva di peso ha concluso che il 39\% dei pazienti operati ha avuto un aumento di peso (specialmente dopo 12 mesi di intervento chirurgico). Tra i fattori che hanno sollevato il problema c'è la scarsa partecipazione alle consultazioni nutrizionali nel periodo preoperatorio, che è culminata nella recidiva di

RC: 98482

Disponibile in: https://www.nucleodoconhecimento.com.br/psicologia-it/follow-uppsicologico 
peso (BARDAL; CECATTO; MEZZOMO, 2016). Gli individui che attraversano un processo di educazione alimentare e consulenza hanno risultati migliori.

La consulenza psicologica e nutrizionale dovrebbe essere prima e dopo l'intervento chirurgico. II rigurgito che di solito colpisce i pazienti che non hanno la corretta abitudine masticatoria è comune ai pazienti che non subiscono alcun tipo di follow-up multidisciplinare (COOPER et al., 1999). Tuttavia, vi è un elevato abbandono nel follow-up nutrizionale postoperatorio, che può portare a complicanze metaboliche e recidiva di peso (MENEGOTTO et al., 2013). Per questi motivi, l'aggiustamento nell'alimentazione nel periodo preoperatorio è di fondamentale importanza, perché, quindi, le nuove abitudini alimentari poste dalle esigenze del periodo postoperatorio saranno più favorevoli alla messa in pratica (RODRIGUES et al., 2017). Aggiustamenti permanenti che includono il comportamento alimentare e le attività fisiche sono necessari per una perdita di peso di successo e per prevenire complicazioni a lungo termine (COSTA, 2013).

Nella valutazione nutrizionale preoperatoria, oltre all'anamnesi nutrizionale completa, vengono eseguiti l'assunzione di cibo, i test di laboratorio, la diagnosi di comorbidità e la storia del peso. È apprezzato per la correzione delle carenze nutrizionali frequentemente identificate. I pazienti obesi a volte subiscono più diete restrittive, e quindi possono essere considerati come un individuo malnutrito (DOLNE; SOUZA, MATTE, 2020). Tuttavia, dovrebbero essere valutate altre questioni relative alla chirurgia, come la disponibilità al cambiamento e la valutazione delle conoscenze generali sulla nutrizione e sulla chirurgia stessa (AILLS et al., 2008). Tali informazioni consentono al professionista di identificare possibili barriere che possono interferire con la perdita di peso o provocare condizioni postoperatorie negative, come l'abuso di alcol e altre droghe. Inoltre, il paziente deve essere preparato per le prime fasi dell'evoluzione della dieta postoperatoria (COSTA, 2013). Pertanto, l'educazione nutrizionale preoperatoria dovrebbe promuovere il miglioramento dei risultati chirurgici.

Disponibile in: https://www.nucleodoconhecimento.com.br/psicologia-it/follow-uppsicologico 
Il supporto psicologico fornito al paziente candidato alla chirurgia bariatrica (pre e postoperatoria) ha alcune finalità specifiche (ROCHA; COSTA, 2012). Tra questi, evidenziamo la conoscenza delle abitudini alimentari, degli stili di vita, del rapporto con il cibo e delle emozioni coinvolte in queste interazioni (KORTCHMAR et al., 2018). Dopo questa conoscenza, lo psicologo diventa in grado di conoscere e tracciare una linea di continuità dal follow-up psicologico postoperatorio, perché le possibilità di raggiungere il successo con il trattamento chirurgico sono più alte (SILVA, 2015). Conoscendo i fattori che possono generare difficoltà in questo processo di adattamento, al paziente viene fornita una maggiore sicurezza e una maggiore attenzione in questi aspetti che lo porrebbero all'interno della gamma di rischio per l'aumento di peso (FAGUNDES; CAREGNATO; SILVEIRA, 2016).

II supporto psicologico, quindi, è un modo per conoscere i fattori che impediscono I'efficacia del trattamento post-bariatrico (MENSORIO, 2013). Dato questo scenario, l'esperienza clinica ha sottolineato che il successo del trattamento chirurgico dell'obesità non dipende solo dalla qualità tecnica della procedura chirurgica. Si tratta di un processo, quindi, che dipende dalla capacità del paziente di adattarsi a un nuovo modello alimentare, un nuovo corpo e un nuovo stile di vita (MAGDALENO JR; CHAIM, CHAIM, TURATO, 2009). Le difficoltà nel cambiare drasticamente la qualità, così come nella quantità di ciò che viene ingerito, possono portare le persone che hanno subito un intervento chirurgico a non perdere peso o, se non tutto, del peso perso (KORTCHMAR et al., 2018). Questo processo può generare la sensazione di fallimento e disperazione, per questo motivo, il supporto psicologico è essenziale.

II mantenimento del peso a lungo termine è essenziale (KULICK; HARK; DEEN, 2010). Un altro punto da valutare è la perdita di peso preoperatoria. Non ci sono prove sufficienti per sostenere questa perdita di peso prima dell'intervento chirurgico, perché quando è fatto, promuove un ritardo inutile per il trattamento del paziente (KIM et al., 2016). Gli studi affermano che una dieta ipocalorica preoperatoria può causare alcune carenze nutrizionali preesistenti, così come la perdita di massa

RC: 98482

Disponibile in: https://www.nucleodoconhecimento.com.br/psicologia-it/follow-uppsicologico 
muscolare può portare il paziente al rischio di malnutrizione, tuttavia, altri studi comprendono che la riduzione del volume addominale ed epatico ha migliori risultati a lungo termine (EDHOLM et al., 2011; THIBAULT et al., 2016). Alla luce del presente articolo, il presente articolo ha come domanda fondamentale: in che modo le strategie tipiche del periodo postoperatorio promuovono una maggiore cura con la dieta e la salute mentale nei pazienti? Per rispondere, è stata effettuata una revisione della letteratura, con l'obiettivo di discutere l'importanza del follow-up psicologico e nutrizionale post-bariatrico.

\section{L'OBESITÀ E LE SUE IMPLICAZIONI}

L'obesità compromette significativamente la qualità della vita e promuove la predisposizione ad altre malattie non trasmissibili, in particolare quelle che danneggiano la salute mentale dell'individuo (MARTINS; MIYAZAKI, 2019). Per questo motivo, l'obesità dovrebbe essere vista come un problema di salute pubblica, considerando che la vita quotidiana delle persone è compromessa.

Al fine di rendere meglio delimitato il contesto di questo studio, le implicazioni della chirurgia bariatrica, il team multidisciplinare che fa parte di questo universo e le cure fornite dallo psicologo e dal nutrizionista dopo l'intervento chirurgico, è necessario comprendere la multifattorialità che coinvolge l'obesità e, di conseguenza, il paziente bariatrico (GORDON; KAIO; SALLET, 2011). Pertanto, inizialmente, è un paziente che ha bisogno di un follow-up psicologico da eseguire continuamente, poiché l'esecuzione di questo tipo di intervento chirurgico richiede un cambiamento di abitudine non solo del paziente, ma anche del gruppo sociale di cui fa parte (KORTCHMAR et al., 2018).

È interessante notare che le questioni familiari e interpersonali, gli aspetti rilevanti della salute mentale, come l'autostima, l'equilibrio affettivo e la percezione della realtà, e infine il follow-up da parte di un team multidisciplinare sono alcuni dei termini che iniziano a integrare la vita della persona obesa, prima o dopo il processo

$\mathrm{RC}: 98482$

Disponibile in: https://www.nucleodoconhecimento.com.br/psicologia-it/follow-uppsicologico 
chirurgico (MARTINS; MIYAZAKI, 2019). Pertanto, è necessario comprendere, in questo scenario, la funzione sociale assunta dallo psicologo e dal nutrizionista e quanto sia essenziale la sua prestazione.

L'aumento della prevalenza di sovrappeso e obesità nel mondo ha preoccupato le agenzie di sanità pubblica, il che ha portato ad un aumento della spesa pubblica. Tuttavia, c'è una giustificazione plausibile: sono necessari sforzi e disponibilità di fondi in numerosi progetti di ricerca che affrontano il tema dell'obesità, poiché aumenta significativamente ogni anno (RASOULI et al., 2007). I numeri non sono tipici della realtà brasiliana, poiché, a causa di determinate abitudini e modelli alimentari, i numeri sono alti in tutto il mondo.

Nazioni come Brasile, Canada e Francia hanno a che fare con un'alta percentuale di individui in sovrappeso (circa il 40\%). Altri paesi, come gli Stati Uniti e la Germania, oltre il $65 \%$ della popolazione è classificata in questo modo. In considerazione di questo scenario, si comprende che questa realtà sociale si riflette nello scenario politico-economico, poiché un sovraccarico viene creato nei sistemi sanitari in modo più significativo, cioè viene investito in cure preventive, consultazioni mediche, farmaci, ricoveri, esami, interventi chirurgici, tra le altre possibilità (MARTINS; MIYAZAKI, 2019). Altri problemi indiretti contribuiscono a questo aumento, come l'assenza di lavoro, il tempo libero perso, le morbilità e la mortalità (MELO, 2011). Lo studio di Kac; Sichieri e Gigante (2007) ha identificato che è meno costoso trattare l'obesità che affrontare le comorbidità che la originano. Tuttavia, sono necessarie misure preventive più efficaci e attuali con il trattamento.

Tali misure preventive, per essere più efficienti, dovrebbero promuovere determinati tipi di pratiche. Tra questi, vi è la necessità di favorire il dialogo tra le molteplici pratiche complementari tra di loro, in particolare quelle che coinvolgono congiuntamente il lavoro dello psicologo e del nutrizionista (GORDON; KAIO; SALLET, 2011). L'obesità dovrebbe essere inserita in un contesto dinamico in cui la malattia esiste, persiste e crea meccanismi che partono da una cattiva

Disponibile in: https://www.nucleodoconhecimento.com.br/psicologia-it/follow-uppsicologico 
alimentazione, basso dispendio energetico, stile di vita sedentario e alto apporto calorico (MARTINS; MIYAZAKI, 2019). È un mezzo che ha anche bisogno di essere trattato e, quindi, la popolazione deve essere interpretata da una prospettiva integrale, considerando che sono inseriti in una cultura del consumo che deve essere riorganizzata. Pertanto, le abitudini che garantiscono il benessere collettivo dovrebbero essere stimolate. In questo contesto, la chirurgia bariatrica emerge e agisce come un'alternativa plausibile.

Tuttavia, la sola bariatria non è in grado di risolvere questo problema di salute pubblica che colpisce la qualità della vita di un certo numero di persone, non solo da una prospettiva locale, ma anche da una prospettiva globale. Pertanto, il monitoraggio psicologico e nutrizionale è di fondamentale importanza e, quindi, si dovrebbe investire in un'assistenza sanitaria che parta da un approccio multidisciplinare (GONÇALVES; KOHLSDORF; PEREZ-NEBRA, 2020). Un attento monitoraggio da parte degli operatori sanitari di più ambiti e della stessa famiglia è essenziale affinché il trattamento a cui è stato sottoposto il paziente sia efficace nel lungo periodo. La chirurgia bariatrica non si limita alla perdita di peso (FANDIÑO et al., 2004). L'obiettivo è soprattutto quello di migliorare lo stato biopsicosociale dell'individuo, tema che permea la qualità della vita (COSTA et al., 2009). Considerando che il successo della chirurgia bariatrica dipende, oltre che dall'equipe multidisciplinare, dalla motivazione del paziente, che implica un cambiamento nei modelli di vita e di consumo (CAMPOS et al., 2016).

\section{LE CARATTERISTICHE DELLA CHIRURGIA BARIATRICA}

L'obesità raggiunge picchi elevati su scala globale, che ha attirato l'attenzione delle autorità, oltre a richiedere molteplici sforzi da parte di professionisti e team sempre più sfaccettati coinvolti nel suo trattamento, che comprende medici, infermieri, psicologi, nutrizionisti e altri professionisti della salute (MARTINS; MIYAZAKI, 2019). Pertanto, cerchiamo alternative che rendano più appropriata la gestione della malattia.

RC: 98482

Disponibile in: https://www.nucleodoconhecimento.com.br/psicologia-it/follow-uppsicologico 
La chirurgia bariatrica, in questo contesto, è considerata da molti pazienti come la via d'uscita più efficace dalla lotta efficace contro l'obesità. Tuttavia, nel periodo postchirurgico, è necessario mantenere il peso, poiché i suoi benefici includono, oltre all'aggiustamento della composizione corporea, una significativa diminuzione delle comorbidità associate (FLORES, 2014). I sintomi depressivi e ansiosi sono significativamente ridotti, così come c'è un miglioramento del funzionamento sessuale, un aumento del livello di attività e un miglioramento integrale della qualità della vita correlata alla salute (FLORES, 2014).

In relazione al contesto brasiliano, il numero di interventi chirurgici legati alla riduzione del peso è gradualmente aumentato, ma in modo espressivo, il che implica la necessità di una maggiore attenzione ai problemi di salute e benessere sociale. A scapito delle critiche e delle controversie associate alla chirurgia bariatrica, si raccomanda che il paziente soddisfi determinati prerequisiti specifici prima di poter essere sottoposto ad esso (GORDON; KAIO; SALLET, 2011). A seconda delle condizioni del paziente, può o non può diventare adatto per la procedura. Uno dei parametri che consentono di prendere questa decisione è il suo follow-up da parte del team multidisciplinare, che implica anche la presenza della famiglia e la forza di volontà del paziente di cambiare abitudini e comportamenti (FANDIÑO et al., 2004). Alcune comorbidità agiscono come fattori aggravanti per il bariatrico non aforego come alternativa alla risoluzione del problema.

Alcuni esempi possono essere citati, come grave pneumopatia, insufficienza renale, marcata lesione miocardica e cirrosi epatica (FANDIÑO et al., 2004). Tuttavia, il bariatrico è generalmente ben accettato e raccomandato. Per quanto riguarda le raccomandazioni per l'intervento bariatrico, spiccano quelle relative alla persona e alle comorbilità. Nei primi anni 1990, il Istituto Superiore di Sanità degli Stati Uniti ha iniziato a sottolineare l'importanza dell'approccio multidisciplinare nel periodo preoperatorio, compresa, in questo processo, la valutazione psicologica prima dell'operazione, essendo una parte essenziale e per il processo decisionale (FLORES, 2014). Anche il Consenso bariatrico brasiliano e il Consiglio federale di

$\mathrm{RC}: 98482$

Disponibile in: https://www.nucleodoconhecimento.com.br/psicologia-it/follow-uppsicologico 
medicina hanno raccomandato questa pratica, ma non senza prima determinare l'importanza della figura di uno psicologo e/o di uno psichiatra nel team multidisciplinare (COUTINHO, 1999).

Come ribadisce il Consiglio federale di medicina, il professionista responsabile, a priori, del trattamento della salute mentale, qui inteso come psicologo e/o psichiatra, fa parte di un team che si assume una responsabilità fondamentale. Ha lo scopo di fornire al paziente una migliore percezione (critica) del loro stato di salute, che contribuisce direttamente a un migliore trattamento adem (ROSA et al., 2020). Durante la valutazione multidisciplinare preoperatoria, oltre a fare una precedente valutazione della storia di salute personale e familiare, l'équipe dovrebbe preoccuparsi, tra le altre cose, della pratica/uso o meno di sostanze psicoattive, della presenza di condizioni psicotiche o mediche, del livello intellettuale e cognitivo che consentano al paziente di essere consapevole dei rischi, successi e cure legate al processo chirurgico a cui sarà sottoposto, come l'assistenza preoperatoria, e anche l'attenzione dovrebbe essere rivolta agli aspetti che influenzano la qualità della vita dei pazienti e delle loro famiglie (FLORES, 2014).

Alla luce di quanto sopra, è necessario discutere la classificazione degli interventi chirurgici bariatrici, che sono: restrittivi o disassortitivi.

Tra i più comuni, c'è la gastrectomia verticale, chiamata anche chirurgia della manica, essendoun intervento chirurgico restrittivo che rimuove gran parte dello stomaco (fondo gastrico e corpo), lasciando uno stomaco residuo a forma di mango (tubo lineare) con capacità volumetrica di $150 \mathrm{ml}$. I pazienti, con questa procedura, sperimentano una riduzione media del $30 \%$ del peso totale nei primi 2 anni (COUTINHO, 1999).

La tecnica Capella, nota come by-pass gastrico in $y$-Roux, consiste inuna modalità che raccoglie la restrizione al disassortimento. Questa è una gastroplastica associata a uno shunt gastrojejunal sotto forma della lettera $Y$ (chiamata di $Y$ di Roux).La

Disponibile in: https://www.nucleodoconhecimento.com.br/psicologia-it/follow-uppsicologico 
procedura riduce le dimensioni dello stomaco in modo che sia in grado di adattarsi a un volume inferiore a $30 \mathrm{ml}$ (MARTINS; MIYAZAKI, 2019). Fa anche connessione con un'ansa intestinale più distale (anastomosi), portando ad una vasta area disaborsotiva, poiché circa 3 metri di snello sono deviati dal transito alimentare (FANDIÑO et al., 2004).

La tecnica Scopinaro, a carattere più disassorbente, è una metodica che consiste nell'applicazione di un "by-pass" biliopancreatico parziale con gastrectomia distale (FANDIÑO et al., 2004). Poiché ha una capacità più malnutritiva ed è più disassorbente, questa tecnica è stata meno eseguita rispetto alle tecniche Sleeve e Capella.

L'obiettivo non è quello di indicare la procedura migliore, ma di elencare le possibilità esistenti e discutere i benefici e i modi che possono far sì che il paziente abbia una migliore qualità della vita, poiché, tra le altre cose, lo scopo principale è quello di contribuire alla riduzione del peso, contribuendo, in parallelo, a migliorare la salute dei pazienti bariatrici.

\section{L'IMPORTANZA DEL FOLLOW-UP NUTRIZIONALE E MENTALE}

\subsection{MONITORAGGIO NUTRIZIONALE}

La chirurgia bariatrica è una procedura che aggiunge risultati positivi al trattamento dell'obesità e delle comorbidità associate. È un argomento che è stato a lungo discusso prevalentemente da medici e nutrizionisti, ma oggi parte di un approccio interdisciplinare, tra cui psicologia, psichiatria, endocrinologia, anestesiologia, cardiologia, fisioterapia, infermieristica ed educazione fisica (FAGUNDES; CAREGNATO; SILVEIRA, 2016). Dato che questi professionisti sono essenziali nel trattamento (TRAVADO et al., 2004).

RC: 98482

Disponibile in: https://www.nucleodoconhecimento.com.br/psicologia-it/follow-uppsicologico 
Uno studio di Silva et al. (2015) ha cercato di identificare il profilo degli individui che hanno scelto di eseguire la chirurgia bariatrica come alternativa al trattamento dell'obesità. Si è concluso che, tra i 100 intervistati, il 49\% ha dichiarato di aver optato per un intervento chirurgico a causa del fallimento di precedenti trattamenti; $39 \%$ a scapito delle malattie associate; e, infine, il $12 \%$ ha optato per la procedura grazie alla sua efficienza e facilità nella perdita di peso in eccesso (SILVA et al., 2015).

Gli obiettivi che hanno motivato i pazienti a cercare la chirurgia bariatrica indicano che l' $87 \%$ ha scelto di desiderare una migliore qualità della vita; $82 \%$ per migliorare la salute; 34\% per fattori estetici; e il 15\% per i fattori sociali (SILVA et al., 2015). I pazienti sottoposti a sei mesi di follow-up nutrizionale preoperatorio hanno avuto una maggiore riduzione di peso rispetto a quelli che hanno smesso di perdere peso preoperatoriamente o riguadagnato il peso perso (KORTCHMAR et al., 2018). In considerazione di questo scenario, si ipotizza che un tempo più lungo di follow-up nutrizionale per i pazienti candidati alla chirurgia bariatrica possa retrocederli, il che potrebbe giustificare i risultati riscontrati dai due studi. In questo senso, le informazioni fornite dal professionista nutrizionista possono promuovere nuove abitudini alimentari, il che aumenta le possibilità di successo dell'intervento chirurgico (ARAGON; ZAMBON, 2021).

La frequenza con cui questi pazienti hanno consultazioni con nutrizionisti è un fattore decisivo per il successo dell'intervento chirurgico. Coloro che si sottopongono a questo follow-up hanno una maggiore perdita di peso in eccesso dopo l'intervento chirurgico (BARDAL; CECCATTO; MEZZOMO, 2016). La recidiva di peso è stata osservata nel 39\% dei pazienti sottoposti a gastroplastica riducente, specialmente a partire da 12 mesi dopo la procedura chirurgica. Per quanto riguarda la perdita di peso preoperatoria, il 74\% dei pazienti ha presentato perdita di peso senza follow-up nutrizionale, il che giustifica la rilevanza di questo professionista nel periodo postoperatorio (BARDAL; CECCATTO; MEZZOMO, 2016). La riduzione del peso nel periodo preoperatorio è benefica, in quanto aumenta l'adesione del paziente ad

$\mathrm{RC}: 98482$

Disponibile in: https://www.nucleodoconhecimento.com.br/psicologia-it/follow-uppsicologico 
abitudini più sane, poiché sarà più condizionato a una dieta adeguata dopo la procedura. Tuttavia, d'altra parte, la riduzione del peso da metodi infondati, come la restrizione dietetica e la dieta ipocalorica senza follow-up nutrizionale, sono alcuni degli aspetti che possono causare situazioni di carenze nutrizionali comuni nei pazienti obesi (RODRIGUES; FARIA, 2020). Questo è il caso della vitamina D e del profilo del ferro nelle donne in età fertile (RODRIGUES et al., 2017).

Un altro fattore che dovrebbe essere considerato nella valutazione è la riduzione della massa muscolare a causa di una grave restrizione calorica. Ciò può causare disturbi della capacità funzionale, ridotta sintesi proteica e diminuzione della competenza immunitaria (BONAZZI et al., 2007). Inoltre, la riduzione del peso preoperatoria si basa sulla facilità transoperatoria per il chirurgo in cui vi è una riduzione del grasso intra-addominale e una riduzione del volume del fegato, che, nella maggior parte dei pazienti che desiderano un intervento chirurgico, è influenzato dalla satatosi epatica. Altri studi comprendono che la riduzione del peso superiore al $9,5 \%$ in più riduce significativamente le complicanze rispetto alla perdita di peso inferiore al $5 \%$ nei pazienti con BMI superiore a $45 \mathrm{~kg} / \mathrm{m}^{2}$. I pazienti con BMI $>50 \mathrm{~kg} / \mathrm{m}^{2}$ possono trarre il massimo beneficio in termini di tecnica chirurgica (THIBAULT et al., 2016).

L'obiettivo del follow-up nutrizionale prima dellintervento chirurgico è quello di aumentare la consapevolezza sull'importanza di aderire a sane abitudini alimentari, essenziali per mantenere la perdita di peso post-chirurgica a lungo termine (GONÇALVES; KOHLSDORF; PEREZ-NEBRA, 2020). In considerazione di questo scenario, la conoscenza dell'alimentazione e della nutrizione negli individui sottoposti e nei candidati alla chirurgia bariatrica solleva alcuni dubbi frequenti, come la possibilità di vomito, la soddisfazione nelle prime 48 ore dopo l'intervento chirurgico, i possibili cambiamenti nel gusto e le condizioni depressive, che rafforza ulteriormente l'importanza delle prestazioni del team multidisciplinare (NISHIYAMA et al., 2007). Uno studio ha rivelato l'impatto espressivo del follow-up interdisciplinare sull'efficacia

RC: 98482

Disponibile in: https://www.nucleodoconhecimento.com.br/psicologia-it/follow-uppsicologico 
della chirurgia bariatrica e ha dimostrato che è di vitale importanza preparare l'individuo prima di eseguire l'intervento chirurgico (MASARI et al., 2012).

Il comportamento alimentare delle persone obese è legato all'ambiente in cui vive l'individuo. Abitudini alimentari inadeguate acquisite durante l'infanzia confermano l'aumento dell'adiposità corporea (NISHIYAMA et al., 2007). Inoltre, c'è un peggioramento della qualità della vita negli individui in sovrappeso, poiché vi è una maggiore possibilità di sviluppare comorbidità associate all'obesità. Pertanto, gli interventi nutrizionali relativi alle pratiche di educazione nutrizionale contribuiscono anche al miglioramento della qualità della vita (BATISTA FILHO; RISSIN, 2003). Uno studio che ha confrontato il comportamento alimentare con la preferenza degli individui nel periodo pre e postoperatorio della chirurgia bariatrica ha rilevato che, dei pazienti in preparazione preoperatoria, il 94\% aveva un "comportamento di pizzicamento". Dei pazienti sottoposti a intervento chirurgico, il $60 \%$ aveva questo stesso comportamento (MACHADO et al., 2008).

Tra i pazienti sottoposti a questa procedura chirurgica, il 33,3\% ha acquisito migliori abitudini alimentari dopo l'intervento chirurgico e il $63 \%$ è rimasto con le stesse abitudini identificate prima dell'intervento chirurgico, il che rafforza ulteriormente l'importanza del follow-up nutrizionale (RODRIGUES et al., 2017). Prima dell'intervento chirurgico, i pazienti avevano una preferenza per gli alimenti ipercalorici, come carboidrati semplici (42\%), dolci (26\%) e snack (14\%) (MACHADO et al., 2008). Tuttavia, dopo l'intervento chirurgico, lo studio ha rilevato che i pazienti cercavano cibi facilmente assumibili, come dolci $(45 \%)$, snack $(22 \%)$, carboidrati $(20 \%)$ e una maggiore preferenza per la birra (7\%) (MACHADO et al., 2008). La frequenza degli episodi di abbuffata da parte dei pazienti è aumentata preoperatoriamente a causa dell'aumento del nervosismo, che era un fattore di squilibrio che stimolava l'assunzione di cibo in eccesso e il $24 \%$ ha riportato la presenza di ansia e / o disturbi dell'umore (depressione).

RC: 98482

Disponibile in: https://www.nucleodoconhecimento.com.br/psicologia-it/follow-uppsicologico 
Gli individui compulsivi obesi hanno una maggiore predisposizione a diventare "pincher" dopo la chirurgia bariatrica, poiché la preferenza per il consumo di dolci e snack è più alta negli obesi compulsivi che negli individui obesi non compulsivi (COLLES; DIXON; O'BRIEN, 2008). Le abitudini di pizzicamento e la compulsione alimentare rimangono o sono migliorate dopo la procedura, il che dimostra ulteriormente l'importanza del follow-up psicologico e / o psichiatrico e nutrizionale nei periodi pre e postoperatorio, poiché abitudini inadeguate possono contribuire all'aumento di peso in questi pazienti. Questi possono essere correlati alla maggiore prevalenza di sintomi gastrointestinali, sindrome da dumping, così come possono causare gravi danni psicologici (FLORES, 2014; MACHADO et al., 2008; GREENBERG; SOGG; PERNA, 2009). L'atto di mangiare è intrinsecamente legato a stimoli interni ed esterni.

Vengono considerati fattori organici, psichici e sociali e, quindi, si percepisce che l'assunzione di cibo va oltre gli aspetti nutrizionali, e quindi riconcilia le motivazioni nascoste legate ai conflitti vissuti e che sono indipendenti dalla sensazione di fame (MACHADO et al., 2008; SOUTO; FERRO-BUCHER, 2006). La limitazione causata dalla chirurgia bariatrica può causare rischi agli individui compulsivi, che possono ostacolare il loro adattamento al nuovo stato alimentare, poiché la capacità gastrica sarà inferiore, e quindi porterà danni se l'individuo continua a nutrirsi in modo errato (BOCCHIERI-RICCIARDI et al., 2006). Gli individui che sono candidati per la chirurgia bariatrica comprendono le dinamiche associate al binge eating. La ricerca di nuove abitudini è un modo per garantire che nuovi disturbi non prevalgano dopo la procedura. Le linee guida nutrizionali devono essere chiarite al paziente.

Argomenti di estrema importanza dovrebbero essere affrontati in modo che si noti il successo post-chirurgico, come la digestione e l'assorbimento dei nutrienti prima e dopo la procedura; linee guida sulla preparazione di piatti più sani; leggere le etichette degli alimenti industrializzati; acquisti sul mercato; e, infine, questi candidati dovrebbero essere istruiti sull'alimentazione durante i primi mesi dopo l'intervento chirurgico (BOCCHIERI-RICCIARDI et al., 2006). L'importanza del nutrizionista

RC: 98482

Disponibile in: https://www.nucleodoconhecimento.com.br/psicologia-it/follow-uppsicologico 
durante l'intero follow-up nel periodo pre e postoperatorio diventa sommaria. Al fine di ottenere risultati positivi. La valutazione preoperatoria deve essere rigorosamente seguita, sulla base di un protocollo di attività precedentemente elaborato. È necessario che il paziente e il team interdisciplinare siano coinvolti e consapevoli dell'importanza di promuovere abitudini alimentari più sane (MASARI et al., 2012).

\subsection{FOLLOW-UP DELLA SALUTE MENTALE}

Gli aspetti psicologici possono influenzare o essere una conseguenza dell'obesità, così come possono essere responsabili dell'aumento di peso o delle difficoltà nel mantenere il peso raggiunto dopo l'intervento chirurgico (VACCARO, 2018). I casi di incapacità di mantenere la perdita di peso sono anche associati alla sensazione di mancanza di preparazione per i cambiamenti necessari. Evitare il fallimento del trattamento bariatrico dipende da un'indagine pre-chirurgica sulla storia del paziente con il cibo, sul ruolo del cibo nella sua vita, così come è necessario indagare e prendersi cura dell'impulso che porta alla voracità del mangiare (EDLER, 2017). La chirurgia è in grado di dimagrire il corpo, tuttavia, alcuni problemi psicologici possono essere trascurati e sono legati al controllo della dieta, all'autostima e alla gestione del mangiare legato all'emozione, il che implica la creazione di un'identità con il corpo, una nuova sfida, perché l'autovalutazione, dopo la perdita di peso, è negativa (FAGUNDES; CAREGNATO; SILVEIRA, 2016).

Questa costruzione negativa è associata all'immagine corporea, all'eccesso di pelle, alla percezione del sé nel nuovo corpo e all'adattamento e alla ricostituzione e all'immagine di sé nel corpo magro (ALEGRÍA; LARSEN, 2013). II follow-up e il supporto psicologico prima e dopo l'intervento chirurgico contribuiscono al miglioramento dei risultati dei pazienti nella loro sfida a perdere peso, a ottenere una maggiore qualità della vita e a ridurre il desiderio di mangiare, essendo questi fattori cruciali per evitare il ripetersi dell'obesità (HOLLYWOOD; OGDEN; PRING, 2012). Ci sono anche associati alla riduzione della fame, alla minima preoccupazione per il

RC: 98482

Disponibile in: https://www.nucleodoconhecimento.com.br/psicologia-it/follow-uppsicologico 
cibo, così come la sensazione di un maggiore controllo per quanto riguarda l'assunzione di cibo.

Uno studio ha rilevato che il tipo di coping adottato dal paziente dopo la chirurgia bariatrica determina o meno il risultato della perdita di peso e che i fattori psicologici e fisiologici si combinano e interagiscono in modo significativo, influenzando così i risultati (la costruzione di un'immagine più positiva sul corpo, ad esempio) (FIGURA et al., 2015).

Gli individui operati possono attraversare un periodo di ristrutturazione emotiva, una fase in cui è essenziale mantenere il follow-up psicologico, attraverso le terapie, per evitare distorsioni cognitive e percezioni sulla realtà deformata. (MAGDALENO JR.; CHAIM; TURATO, 2008). In considerazione di questo scenario, si richiama l'attenzione sulla necessità di un trattamento psicologico individualizzato nel periodo pre e post-chirurgico. È legato all'identificazione e al trattamento della struttura mentale di ciascun paziente, mirando, con queste cure, ad evitare ulteriori complicazioni dopo l'intervento chirurgico (GORDON; KAIO; SALLET, 2011). Le esperienze fisiche, psicologiche, sociali e cliniche dei pazienti sottoposti a chirurgia bariatrica influenzano il processo di trattamento. Tra quelli che più interessano questi soggetti ci sono: cambiamenti fisici e sfide successive, esperienze psicologiche, supporto sociale ed esperienze cliniche (LIU; IRWIN, 2017).

C'è un miglioramento delle condizioni psicologiche, fisiche e sociali, tuttavia, c'è una continua preoccupazione per possibili eventi imprevisti tipici dei risultati della chirurgia e l'importanza di sostenere i servizi sanitari raccomandati per il periodo post-chirurgico, incluso, in questo processo, il supporto psicologico, poiché i pazienti devono imparare come affrontare i cambiamenti nel corpo, le relazioni sociali e la costruzione di una nuova identità (LIU; IRWIN, 2017). Anche i parenti o i caregiver del paziente bariatrico hanno bisogno di supporto psicologico (SPRENGEL, 2015), poiché il supporto sociale a tutti i soggetti coinvolti implica una migliore adesione a comportamenti di salute più sani, come l'attività fisica, un'alimentazione più sana, la

RC: 98482

Disponibile in: https://www.nucleodoconhecimento.com.br/psicologia-it/follow-uppsicologico 
riduzione delle situazioni di stress (MARTINS; MIYAZAKI, 2019). Pertanto, viene promosso il miglioramento della salute, compresa la salute mentale e la qualità della vita (ABREU RODRIGUES; SEIDL, 2015).

Tuttavia, l'efficacia del trattamento richiede alcune cure, come il tempo di preparazione psicologica prima e dopo l'intervento chirurgico, sia per il paziente che per i coniugi e i familiari, poiché sono loro che li accompagneranno durante il trattamento pre e post-bariatrico (DELAPRIA, 2019). La motivazione e il supporto a lungo termine del paziente dovrebbero essere stimolati nel processo di follow-up psicologico (MARCHESINI, 2010). In questo viaggio, includere sia la famiglia che gli amici nel processo preoperatorio è di fondamentale importanza, oltre a garantire la chiarezza del paziente per quanto riguarda i guadagni e le perdite coinvolti nella procedura (VACCARO, 2018). II trattamento, quindi, richiede l'esecuzione di un team multidisciplinare. Questo dovrebbe comprendere la soggettività di ciascun caso, prevedendo e accompagnando quei fattori che, direttamente o indirettamente, possono influenzare il successo o il fallimento del processo, il che implica il ridimensionamento di determinate strategie per raggiungere il successo (MARCELINO; PATRÍCIO, 2011).

La chirurgia bariatrica, infatti, offre una migliore qualità della vita al paziente, oltre a promuovere il miglioramento della socializzazione dovuta alla perdita di peso, tuttavia, va sottolineato che, in questo processo di trattamento, il paziente dovrà comunque affrontare problemi psichici e nutrizionali per raggiungere la qualità di vita desiderata (DELAPRIA, 2019). II supporto è essenziale affinché i cambiamenti nel comportamento alimentare e nello stile di vita possano essere resi possibili, così come i cambiamenti del corpo, sociali e dell'identità (VACCARO, 2018). II mantenimento e la continuità del peso dovuti al trattamento chirurgico dipendono dall'azione, dalla consapevolezza e dall'impegno del paziente, che può essere difficile e irrealizzabile senza un follow-up psicologico e nutrizionale.

RC: 98482

Disponibile in: https://www.nucleodoconhecimento.com.br/psicologia-it/follow-uppsicologico 
Il paziente stesso deve rendersi conto di essere l'agente trasformante. La chirurgia dimagrisisce, ma il corpo magro dipende da cambiamenti interni, una richiesta che va oltre lo stomaco. L'adattamento alle nuove dimensioni del corpo e l'adattamento nelle relazioni sociali, così come la costruzione di una nuova identità e la disconnessione dell'impulso a mangiare sono problemi che possono sorgere dopo l'intervento chirurgico, e quindi richiedono maggiore attenzione e cura (GORDON; KAIO; SALLET, 2011). La psicoterapia può contribuire alla mente seguendo il processo di dimagrimento del corpo.

\section{CONSIDERAZIONI FINALI}

Questo studio mirava a dimostrare l'importanza del follow-up nutrizionale e mentale prima e dopo la chirurgia bariatrica. L'evidenza indica che il ruolo svolto dallo psicologo nel team multidisciplinare, specialmente nel periodo postoperatorio dei pazienti bariatrici, per quanto riguarda il controllo e / o la riduzione dell'ansia e dei sintomi depressivi e di altri cambiamenti psicopatologici che danneggiano la salute mentale, è essenziale, il che rende fondamentale il dibattito sul ruolo degli psicologi e degli altri professionisti coinvolti. La rilevanza di questi professionisti è legata al fatto che un'azione multidisciplinare farà abituare il paziente sottoposto a bariatria ad un nuovo tenore di vita (abitudini alimentari, rapporto con il cibo e il corpo) e, in questo processo, il follow-up psicologico durante il periodo pre e postoperatorio è rilevante per evitare problemi come l'aumento di peso.

La valutazione psicologica dei pazienti bariatrici è rilevante, perché, in questo modo, è possibile comprendere questo paziente in modo olistico e, quindi, viene promossa una riduzione delle complicanze mentali da un intervento chirurgico che può compromettere la qualità della vita del paziente. La necessità di una valutazione psicologica approfondita diventa sommaria. II paziente deve essere studiato e istruito in modo che i cambiamenti successivi alla chirurgia siano efficaci e visualizzati a lungo termine. Le indicazioni e le controindicazioni legate alla chirurgia bariatrica dovrebbero rispettare la valutazione del team multidisciplinare, considerando che, a

RC: 98482

Disponibile in: https://www.nucleodoconhecimento.com.br/psicologia-it/follow-uppsicologico 
causa delle raccolte e delle pressioni quotidiane, i problemi che colpiscono la salute mentale, come i disturbi d'ansia, come i disturbi d'ansia, tra cui spiccano la fobia sociale, l'ansia generalizzata e il disturbo di panico, in relazione ai disturbi dell'umore, il crescente numero di persone affette da depressione è preoccupante.

Tali disturbi sono diventati frequenti e affliggono la vita di molte persone. È nel bel mezzo di questo processo, in un certo senso, turbolento, che si trova il paziente postbariatrico, questo deve fare i conti, quasi sempre, con un carico emotivo oltre il sopportabile, che finisce per creare disturbi inaspettati associati all'ansia. In questo senso, lo psicologo svolge un ruolo molto importante sin dall'inizio del processo chirurgico, ma le sue prestazioni non sono limitate a lui. Pertanto, il suo contributo nel periodo postoperatorio è essenziale.

\section{RIFERIMENTI}

ABREU-RODRIGUES, M.; SEIDL, E. M. F. Apoio social e reganho de peso póscirurgia bariátrica: estudo de caso sobre intervenção com cuidador. Temas em Psicologia, v. 23, n. 4, p. 1003-1016, 2015.

AILLS, L. et al. ASMBS allied health nutritional guidelines for the surgical weight loss patient: surgery for obesity and related diseases. Surg Obes Relat Dis., v. 4 , n. 5 , p. $73-108,2008$.

ALEGRÍA, C. A.; LARSEN, B. "That's who I am: A fat person in a thin body": Weight loss, negative self-evaluation, and mitigating strategies following weight loss surgery. Journal of the American Association of Nurse Practitioners, v. 27, n. 3, p. 137144,2013

ARAGÃO, C. N.; ZAMBON, E. A avaliação psicológica para cirurgia bariátrica - uma análise teórica e prática. Rumos da inFormação, v. 2, n. 1, p. 63-76, 2021.

RC: 98482

Disponibile in: https://www.nucleodoconhecimento.com.br/psicologia-it/follow-uppsicologico 
BARDAL, A. G.; CECCATTO, V.; MEZZOMO, T. R. Fatores de risco para recidiva de peso no pós-operatório tardio de cirurgia bariátrica. Scientia médica, v. 26, n. 4, p. ID24224-ID24224, 2016.

BATISTA FILHO, M.; RISSIN, A. A transição nutricional no Brasil: tendências regionais e temporais. Cadernos de saúde pública, v. 19, p. S181-S191, 2003.

BOCCHIERI-RICCIARDI, L. E. et al. Pre-surgery binge eating status: effect on eating behavior and weight outcome after gastric bypass. Obesity surgery, v. 16, n. 9, p. 1198-1204, 2006.

BONAZZI, C. L. et al. A intervençâo nutricional no pré e pós operatório da cirurgia bariátrica. RBONE-Revista Brasileira de Obesidade, Nutrição e Emagrecimento, v. 1 , n. 5 , p. 59-69, 2007.

BRASIL. Ministério da Saúde. Vigitel 2016: vigilância de fatores de risco e proteção para doenças crônicas por inquérito telefônico. Brasília: Ministério da Saúde, 2017.

BUCHWALD, $\mathrm{H}$. et al. Bariatric surgery: a systematic review and meta-analysis. Jama, v. 292, n. 14, p. 1724-1737, 2004.

CAMPOS, J. et al. O papel da cirurgia metabólica para tratamento de pacientes com obesidade grau ie diabete tipo 2 não controlados clinicamente. ABCD: Arquivos Brasileiros de Cirurgia Digestiva (São Paulo), v. 29, p. 102-106, 2016.

COLLES, S. L.; DIXON, J. B.; O'BRIEN, P. E. Loss of control is central to psychological disturbance associated with binge eating disorder. Obesity, v. 16, n. 3 , p. $608-614,2008$.

COOPER, P. L. et al. Nutritional consequences of modified vertical gastroplasty in obese subjects. International journal of obesity, v. 23, n. 4, p. 382-388, 1999.

$\mathrm{RC}: 98482$

Disponibile in: https://www.nucleodoconhecimento.com.br/psicologia-it/follow-uppsicologico 
CostA, A. C. C. et al. Obesidade em pacientes candidatos a cirurgia bariátrica. Acta Paulista de enfermagem, v. 22, p. 55-59, 2009.

COSTA, D. Eficiência do acompanhamento nutricional no pré e pós-operatório da cirurgia bariátrica. RBONE-Revista Brasileira de Obesidade, Nutrição e Emagrecimento, v. 7, n. 39, 2013.

COUTINHO, W. Consenso latino-americano de obesidade. Arquivos Brasileiros de Endocrinologia \& Metabologia, v. 43, n. 1, p. 21-67, 1999.

DELAPRIA, A. M. T. A importância do acompanhamento psicológico no pré e pósoperatório da cirurgia bariátrica. Revista UNINGÁ, v. 56, n. S1, p. 78-88, 2019.

DOLNE, F.; SOUZA, J. S. M. de.; MATTE, J. Atuação da enfermagem na assistência prestada ao paciente obeso mórbido no pós-operatório de cirurgia bariátrica. RBONE-Revista Brasileira De Obesidade, Nutrição E Emagrecimento, v. 14, n. 87 , p. $550-559,2020$.

EDHOLM, D. et al. Preoperative 4-week low-calorie diet reduces liver volume and intrahepatic fat and facilitates laparoscopic gastric bypass in morbidly obese. Obesity surgery, v. 21, n. 3, p. 345-350, 2011.

EDLER, S. Tempos Compulsivos: a busca desenfreada pelo prazer. Rio de Janeiro: Casa da Palavra, 2017. 176 pgns.

FAGUNDES, M. A. B. G.; CAREGNATO, R. C. A.; SILVEIRA, L. M. de. O. B. Variáveis psicológicas associadas à cirurgia bariátrica. Aletheia, v. 49, n. 2, p. 4754, 2016.

FANDIÑO, J. et al. Cirurgia bariátrica: aspectos clínico-cirúrgicos e psiquiátricos. Revista de psiquiatria do Rio Grande do Sul, v. 26, n. 1, p. 47-51, 2004. 
FIGURA, A. et al. Determinants of weight loss following laparoscopic sleeve gastrectomy: the role of psychological burden, coping style, and motivation to undergo surgery. Journal of obesity, v. 2015, p. 1-10, 2015.

FLORES, C. A. Avaliação psicológica para cirurgia bariátrica: práticas atuais. ABCD: Arquivos Brasileiros de Cirurgia Digestiva (São Paulo), v. 27, p. 59-62, 2014.

GONÇALVES, S. J. B. G.; KOHLSDORF, M.; PEREZ-NEBRA, A. R. Adesão ao pósoperatório em cirurgia bariátrica: análise sistemática da literatura brasileira. Psicologia Argumento, v. 38, n. 102, p. 626-646, 2020.

GORDON, P. C.; KAIO, G. H.; SALLET, P. C. Aspectos do acompanhamento psiquiátrico de pacientes obesos sob tratamento bariátrico: revisão. Archives of Clinical Psychiatry (São Paulo), v. 38, p. 148-154, 2011.

GREENBERG, I.; SOGG, S.; PERNA, F. M. Behavioral and psychological care in weight loss surgery: best practice update. Obesity, v. 17, n. 5, p. 880-884, 2009.

HOLLYWOOD, A.; OGDEN, J.; PRING, C. The impact of a bariatric rehabilitation service on weight loss and psychological adjustment-study protocol. BMC Public Health, v. 12, n. 1, p. 1-5, 2012.

KAC, G.; SICHIERI, R.; GIGANTE, D. P. Epidemiologia nutricional. São Paulo: Editora Fiocruz, 2007. 580 pgns.

$\mathrm{KIM}$, J. et al. ASMBS updated position statement on insurance mandated preoperative weight loss requirements. Surgery for Obesity and Related Diseases, v. 12, n. 5, p. 955-959, 2016.

KORTCHMAR, E. et al. Reganho de peso após a cirurgia bariátrica: um enfoque da fenomenologia social. Acta Paulista de Enfermagem, v. 31, n. 4, p. 417-422, 2018. 
KULICK, D.; HARK, L.; DEEN, D. The bariatric surgery patient: a growing role for registered dietitians. Journal of the American Dietetic Association, v. 110, n. 4, p. 593-599, 2010.

LIU, R. H.; IRWIN, J. D. Understanding the post-surgical bariatric experiences of patients two or more years after surgery. Quality of Life Research, v. 26, n. 11, p. 3157-3168, 2017.

MACHADO, C. E. et al. Compulsão alimentar antes e após a cirurgia bariátrica. ABCD: Arquivos Brasileiros de Cirurgia Digestiva (São Paulo), v. 21, p. 185-191, 2008.

MAGDALENO JR, R.; CHAIM, E. A.; TURATO, E. R. Características psicológicas de pacientes submetidos à cirurgia bariátrica. Revista de Psiquiatria do Rio Grande do Sul, v. 31, p. 73-78, 2009.

MAGDALENO JR, R.; CHAIM, E. A.; TURATO, E. R. Psychological characteristics of patients submitted to bariatric surgery. Revista de Psiquiatria do Rio Grande do Sul, v. 31, p. 73-78, 2009.

MARCELINO, L. F.; PATRICIO, Z. M. A complexidade da obesidade e o processo de viver após a cirurgia bariátrica: uma questão de saúde coletiva. Revista Ciência e Saúde Coletiva, v. 16, n. 12, p. 4767-4776, 2011.

MARCHESINI, S. D. Late psychological follow-up on patients submitted to bariatric surgery. ABCD: Arquivos Brasileiros de Cirurgia Digestiva, v. 23, n. 2, p. 108113, 2010.

MARTINS, C. E. P.; MIYAZAKI, E. T. A presença do psicólogo na equipe multidisciplinar e a ansiedade pós-cirurgia bariátrica. Revista Campo do Saber, v. 5, n. 2, p. 77-90, 2019

RC: 98482

Disponibile in: https://www.nucleodoconhecimento.com.br/psicologia-it/follow-uppsicologico 
MASARI, S. P. et al. Preparação multidisciplinar pré-cirúrgia bariátrica na visão do cliente. RBONE-Revista Brasileira de Obesidade, Nutrição e Emagrecimento, v. 6, n. 32, p. 1-13, 2012.

MELO, M. E. Os Números da Obesidade no Brasil: VIGITEL 2009 e POF 20082009. 2011. Disponível em: https://www.saudedireta.com.br/docsupload/1340368729Obesidade\%20no\%20Brasil \%20VIGITEL\%202009\%20POF2008_09\%20\%20II.pdf. Acesso em: 02 ago. 2021.

MENEGOTTO, A. L. S. et al. Avaliação da frequência em consultas nutricionais dos pacientes após cirurgia bariátrica. ABCD: Arquivos Brasileiros de Cirurgia Digestiva (São Paulo), v. 26, p. 117-119, 2013.

MENSORIO, M. S. Análise de estratégias de enfrentamento, ansiedade e hábitos em pacientes elegíveis à cirurgia bariátrica, com e sem acompanhamento psicológico. 2013. 159f. Dissertação (Mestrado em Processos de Desenvolvimento Humano e Saúde) - Universidade de Brasília, Brasília, DF, 2013.

NISHIYAMA, M. F. et al. Avaliação do nível de conhecimento e aderência da conduta nutricional em pacientes submetidos e candidatos à cirurgia bariátrica. Arquivos de Ciências da Saúde da UNIPAR, v. 11, n. 2, p. 89-98, 2007.

RASOULI, N. et al. Ectopic fat accumulation and metabolic syndrome. Diabetes, Obesity and Metabolism, v. 9, n. 1, p. 1-10, 2007.

ROCHA, C.; COSTA, E. Aspectos psicológicos na obesidade mórbida: Avaliação dos níveis de ansiedade, depressão e do auto-conceito em obesos que vão ser submetidos à cirurgia bariátrica. Analise psicológica, v. 30, n. 4, p. 451-466, 2012.

RC: 98482

Disponibile in: https://www.nucleodoconhecimento.com.br/psicologia-it/follow-uppsicologico 
RODRIGUES, G. et al. Acompanhamento nutricional no pré-operatório de cirurgia bariátrica: tempo de seguimento versus redução de peso. Revista PsicoFAE: Pluralidades em Saúde Mental, v. 6, n. 2, p. 97-112, 2017.

RODRIGUES, J. O.; FARIA, H. M. C. Os aspectos psicossociais da cirurgia bariátrica: do pré ao pós-operatório. Cadernos de Psicologia, v. 2, n. 4, p. 551-569, 2020.

ROSA, T. M. F. et al. Atendimento nutricional em grupo de pacientes candidatos a cirurgia bariátrica: repercussões no consumo alimentar. RBONE-Revista Brasileira De Obesidade, Nutrição E Emagrecimento, v. 14, n. 87, p. 690-698, 2020.

SILVA, M. A da. Emagrecimento induzido por cirurgia: ação do psicólogo após a cirurgia bariátrica. Bol. Psicol., v. 65, n. 143, p. 243-246, 2015.

SILVA, P. T. da. et al. Perfil de pacientes que buscam a cirurgia bariátrica. ABCD: Arquivos Brasileiros de Cirurgia Digestiva (São Paulo), v. 28, p. 270-273, 2015.

SOCIEDADE BRASILEIRA DE CIRURGIA BARIÁTRICA E METABÓLICA (SBCBM). 2017. Disponível em: http://www.sbcbm.org.br/. Acesso em: 02 ago. 2021.

SOUTO, S.; FERRO-BUCHER, J. S. N. Práticas indiscriminadas de dietas de emagrecimento e o desenvolvimento de transtornos alimentares. Revista de Nutrição, v. 19, p. 693-704, 2006.

SPRENGEL, A. L. Cirurgia Bariátrica: Manual de instruções para pacientes e familiares. São Paulo: M. Books do Brasil, 2015. 96 pgns.

THIBAULT, R. et al. Twelve key nutritional issues in bariatric surgery. Clinical nutrition, v. 35, n. 1, p. 12-17, 2016.

RC: 98482

Disponibile in: https://www.nucleodoconhecimento.com.br/psicologia-it/follow-uppsicologico 
TRAVADO, L. et al. Abordagem psicológica da obesidade mórbida: Caracterização e apresentação do protocolo de avaliação psicológica. Análise psicológica, v. 22, n. 3, p. 533-550, 2004.

VACCARO, R. V. Acompanhamento psicológico e manutenção da perda de peso após a cirurgia bariátrica do tipo Bypass. 2018. 81f. Dissertação (Mestrado em Psicologia Clínica) - Pontifícia Universidade Católica de São Paulo, São Paulo, SP, 2018.

Inserito: Ottobre 2021.

Approvato: Ottobre 2021.

RC: 98482

Disponibile in: https://www.nucleodoconhecimento.com.br/psicologia-it/follow-uppsicologico 